\title{
Valoración del eje hipotálamo-hipofisario en la fase precoz del traumatismo craneoencefálico grave
}

\author{
MANUEL PÉREZ-ALÉa , JUAN MANUEL FLORES-CORDERO', M. DOLORES RINCÓN-FERRARI \\ SUSANA GARCÍA-GÓMEZ ${ }^{\mathrm{b}}$, JOSÉ IGNACIO SÁNCHEZ-OLMEDO ${ }^{\mathrm{a}}$, FRANCISCO MURILLO-CABEZAS ${ }^{\mathrm{b}}$ \\ Y ALFONSO LEAL-CERRO ${ }^{\circ}$
}

aServicio de Medicina Intensiva. Hospital General Básico de la Defensa de San Fernando. San Fernando. Cádiz. España. bServicio de Cuidados Críticos y Urgencias. Hospital Universitario Virgen del Rocío. Sevilla. España.

'Servicio de Endocrinología. Hospital Universitario Virgen del Rocío. Sevilla. España.

Objetivo. Analizar las características de la función hipofisaria en la fase inicial de pacientes críticos con traumatismo craneoencefálico grave (TCEG).

Diseño. Estudio observacional, prospectivo. La función hipofisaria se evaluó siempre el tercer día tras el TCEG.

Ámbito. Unidad de cuidados intensivos (UCI) neurotraumatológica de un hospital universitario.

Pacientes. Se incluyó a 136 pacientes con TCEG (intervalo, 16-65 años), durante un período de 2 años y 9 meses y estancia en $\mathrm{UCl}$ mayor de $48 \mathrm{~h}$.

Intervención. Ninguna.

Medidas y datos recopilados. Se recogieron durante las primeras $72 \mathrm{~h}$ tras el traumatismo: variables demográficas, severidad de la lesión, parámetros de neuromonitorización, lesiones cerebrales secundarias, uso de fármacos vasoactivos y el tipo de traumatismo craneoencefálico (TCE) acorde a los hallazgos encontrados en la tomografía computarizada (TC). La evaluación de la función hipofisaria se determinó por medición de hormonas hipofisarias y las de los órganos diana, con la excepción de la función somatotropa, que se evaluó midiendo las concentraciones séricas basales de insulin-like growth factor-1 (IGF-1).

Resultados. Se observó disfunción hipofisaria en $101(74,2 \%)$ pacientes; $79(58 \%)$ pacientes tenían afectado solamente un eje hipofisario, la afectación de ejes es la siguiente: gonadotropo, el $63,7 \%$ (87 pacientes); tirotropo, el 8,8\% (12 pa-

Correspondencia: Dr. M. Pérez Alé.

Cobre, 5. 41710 Utrera. Sevilla. España.

Correo electrónico: manuel.manuelale@gmail.com

Manuscrito aceptado el 5-5-2008 cientes), y corticotropo, el $0,7 \%$ ( 1 paciente). Se observaron concentraciones plasmáticas bajas de IGF-1 acorde a la edad en $90(66,7 \%)$ pacientes, aunque sólo 26 de ellos mostraron un valor menor de $90 \mathrm{ng} / \mathrm{ml}$.

Conclusiones. Nuestros datos muestran que la disfunción hipofisaria ocurre precozmente y con gran frecuencia tras un TCEG, aunque el significado real de estos hallazgos están aún por determinar.

PALABRAS CLAVE: Hormonas hipofisarias. Hipopituitarismo. Traumatismo craneoencefálico grave.

\section{ASSESSMENT OF HYPOTHALAMO-PITUITARY AXIS IN EARLY PHASE OF SEVERE CRANIOENCEPHALIC BRAIN INJURY}

Objective. To assess early pituitary function in a sequential cohort of critical care patients after severe traumatic brain injury (TBI).

Design. This was a prospective observational study. The pituitary function was always tested on the third day after TBI.

Setting. Neurocritical intensive care unit (ICU) in a University hospital.

Patients. A total of 136 adult patients with severe TBI (range, 16-65 years) enrolled over a 2 year and 9 month period having a stay in the ICU treated than 48 hours.

Intervention. None.

Measurements and data collected. The following data were recorded within the first 72 hours after injury: demographic variables, injury severity, neuromonitoring data, systemic secondary brain insults, use of vasoactive drugs and type of TBI according to the computerized tomography 
(CT) scan findings. Pituitary function was evaluated by measurement of both the pituitary and target organ hormones, with the exception of the somatotrophic function, which was assessed by measurement of basal serum values of insulinlike growth factor-I (IGF-I).

Results. Pituitary dysfunction was observed in 101 patients $(74.2 \%)$. Seventy-nine patients $(58 \%)$ had impairment of only one pituitary axis, the axes being affected as follows: gonadotropic $63.7 \%$ (87 patients), thyrotropic 8.8\% (12 patients) and corticotropic $0.7 \%$ ( 1 patient). Low IGF-1 plasmatic levels in accordance to the patient's age were observed in 90 patients $(66.7 \%)$. However, only 26 of them had a value below $90 \mathrm{ng} / \mathrm{ml}$.

Conclusions. Our data show that pituitary dysfunction occurs early and with high frequency after severe TBI, but the real significance of these findings still needs to be elucidated.

KEY WORDS: Pituitary hormones. Hypopituitarism. Traumatic brain injury.

\section{INTRODUCCIÓN}

Los traumatismos representan un grave problema de salud pública en los países industrializados y conllevan una elevada tasa de morbilidad e incapacidad, con un elevado coste social, sanitario y, por supuesto, económico ${ }^{1}$. Entre éstos, el traumatismo craneoencefálico (TCE) es la mayor causa de incapacidad y mortalidad en el segmento más joven de la población. Hasta en un $70 \%$ de los traumatismos el TCE es la causa de muerte del paciente. Igualmente el grado de recuperación funcional está determinado, fundamentalmente, por el grado de lesión neurológica que se presente $^{2}$. El progreso en la atención inmediata quirúrgica e intensiva ha conseguido salvar de la muerte a un gran número de personas, pero está generando un incremento en la gravedad de las secuelas. Tras el traumatismo craneoencefálico grave (TCEG), se originan en el sistema nervioso central cambios estructurales y fisiológicos que pueden comprometer varias funciones cerebrales. Factores como la naturaleza, la extensión, el lugar del daño cerebral, el tipo de traumatismo -abierto o cerrado-y la dominancia hemisférica se relacionan con los diferentes efectos del daño cerebral ${ }^{3}$. Entre los supervivientes del TCEG, un considerable número de ellos quedan con importantes secuelas que impiden el retorno a sus actividades anteriores o imposibilitan la integración académica, profesional y social, y la calidad de vida es altamente dependiente del grado de afectación neuropsicológica. Las secuelas intelectuales de los TCE dificultan la reintegración social y ocupacional en mayor grado que las de tipo físico, ya que después de un TCE moderado o grave todos los pacientes presentan alteraciones cognitivas, de memoria, de coordinación motora fina y del lenguaje. Todo esto supone, como hemos mencionado anteriormente, un coste humano difícil de estimar y un elevado coste económico $^{4}$

Las secuelas neuropsicológicas (problemas de memoria, irritabilidad, lentitud, falta de concentración, fatiga, etc. $)^{3,5}$ y las disfunciones neuroendocrinas (anorexia, letargia, náuseas, pérdida de peso, crisis comiciales, trastornos de presión arterial, desregulación de la temperatura corporal, etc.) ${ }^{6,7}$ también influyen en la morbilidad de estos pacientes. El hipopituitarismo postraumático es conocido desde hace unos 80 años, aunque inicialmente se consideraba como un hecho excepcional ${ }^{4}$. Recientemente se ha demostrado que el TCE es una causa frecuente de alteración de la función hipotálamo-hipofisaria, que contribuye al retraso y/o a la torpe recuperación de los pacientes con $\mathrm{TCE}^{7-11}$.

Estudios encaminados, por una parte, a conocer realmente la incidencia y los tipos de déficit hormonales hipofisarios en su fase aguda y a lo largo de su evolución y, por otra, a establecer qué características iniciales del TCE se comportan como factores de riesgo para desarrollar hipopituitarismo son necesarios para definir qué pacientes podrían ser tributarios en un futuro de estudios controlados, encaminados a valorar los beneficios de la terapia hormonal sustitutiva.

En el presente trabajo se ha valorado la función hipofisaria en la fase inicial del TCEG.

\section{PACIENTES Y MÉTODOS}

\section{Diseño}

Estudio observacional, prospectivo, realizado durante un período de 2 años y 9 meses ( 1 de marzo de 2003-31 de diciembre de 2005), en el Hospital Universitario Virgen del Rocío de Sevilla. El Comité de Ética del hospital aprobó el estudio y se obtuvo el consentimiento informado de los familiares de los pacientes.

\section{Muestra: sujetos de estudio}

Se incluyó a 136 pacientes, ingresados en la unidad de cuidados intensivos (UCI) de neurocríticos, diagnosticados de TCEG, definido por un nivel de conciencia, medido por la Glasgow Coma Scale (GCS), entre 4 y 8 puntos, tras una reanimación adecuada, en el área extrahospitalaria o de urgencias. La edad de los pacientes incluidos se encontraba entre los 16 y 65 años, ambos inclusive. Los pacientes debían llevar ingresados al menos $48 \mathrm{~h}$, y era necesario el consentimiento informado firmado por los familiares o por el representante legal. Los criterios de exclusión aplicados fueron: TCE con GCS de 3 puntos, midriasis bilateral arreactiva u otros signos clínicos y/o complementarios indicativos de muerte cerebral, tras la reanimación adecuada, edad $<16$ años o $>65$ años, no tener firmado el consentimiento informado, mujeres embarazadas y la ingesta previa de corticoides. 
Durante el período de estudio el manejo de los pacientes incluidos fue el habitual en nuestra unidad. Se realizó a todos los pacientes intubación endotraqueal en la escena del accidente o en el área de urgencias y recibieron ventilación mecánica. La sedación se realizó con midazolam. En nuestra UCI, se monitoriza la presión intracraneal (PIC) a los pacientes con TCEG y el tratamiento se basa en las recomendaciones de la Brain Trauma Foundation Guideline $^{12}$, con el objetivo de mantener la PIC $<20$ $\mathrm{mmHg}$ y la presión de perfusión cerebral (PPC) $\geq 70$ $\mathrm{mmHg}$. La monitorización general siempre incluyó registro continuo de presión venosa central (PVC) y presión arterial (PA). El objetivo del manejo de fluidos y electrolitos es establecer y mantener una situación de normovolemia y una concentración sérica de sodio de aproximadamente $145 \mathrm{mmol} / \mathrm{l}$. Ningún paciente recibió corticoides o furosemida antes de la realización del estudio.

\section{Variables analizadas}

Durante su estancia en UCI se recogieron las siguientes variables: hipoxemia e hipotensión prehospitalaria, edad, sexo, índice de masa corporal (IMC), mecanismo lesional, áreas corporales lesionadas, alteración pupilar (se recogió el tamaño de las pupilas, su simetría y la reacción pupilar a la luz) y mortalidad. En las mujeres se recogió su historia menstrual, se registró la administración de corticoides por vía parenteral en la asistencia prehospitalaria, GCS, Injury Severity Score (ISS), Acute Physiology and Chronic Health Evaluation II (APACHE II). Estas escalas de gravedad se recogieron en el tercer día de evolución (día de extracción de sangre para el estudio hormonal). Datos de neuromonitorización: PIC y PPC, que se recogieron cada hora, y se anotaban los cambios ocasionales indicativos de eventos altamente críticos. Aunque el umbral crítico de PIC y PPC en cada paciente es específico y variable durante su evolución, un valor de $20 \mathrm{mmHg}$ para la PIC y 70 mmHg para la PPC se suelen aceptar como el umbral para definir la hipertensión endocraneal (HEC) e hipoperfusión cerebral. En el presente estudio se estableció que el paciente tenía HEC e hipoperfusión cerebral cuando presentaba al menos dos registros diarios de PIC mayores de $20 \mathrm{mmHg}$ y de PPC menor de $70 \mathrm{mmHg}$, respectivamente. Otros valores analizados fueron los factores sistémicos favorecedores de lesión cerebral secundaria: hipoxia $\left(\mathrm{PaO}_{2} / \mathrm{FiO}_{2}<250\right)$, hipotensión arterial (presión arterial sistólica [PAS] $<90 \mathrm{mmHg}$ que no requiere reposición extra de volumen ni manejo de fármacos vasoactivos), shock (PAS $<90 \mathrm{mmHg}$ que requiere reposición de volumen extra o la instauración de fármacos vasoactivos), hiperglucemias (glucemias $>$ $180 \mathrm{mg}$ ), hipertermia (temperatura central $>38^{\circ} \mathrm{C}$ ) y anemia (hemoglobina $<13 \mathrm{~g} / \mathrm{dl}$ en varones y 11,5 $\mathrm{g} / \mathrm{dl}$ en mujeres); se registró a los pacientes que necesitaron administración de dopamina y/o noradrenalina. Se definió el tipo de lesión craneal según la Traumatic Coma Data Bank (TCDB) ${ }^{13}$.

\section{Evaluación hormonal}

La valoración hormonal se realizó siempre el tercer día tras la lesión traumática. Las muestras de sangre se obtuvieron por la mañana (8.00-10.00) en todos los pacientes. Todas las determinaciones se realizaron mediante técnicas comercialmente disponibles. Los valores de cortisol, lutropina ( $\mathrm{LH}$ ), folitropina (FSH), tirotropina (TSH), prolactina (PRL), T4 libre, estradiol -en mujeres- y testosterona total -en varonesse determinaron mediante fluoroinmunoanálisis (Autodelfia, Wallac, Inc., Turku, Finlandia). Las concentraciones basales del factor de crecimiento similar a la insulina 1 (IGF-1) y corticotropina (ACTH) se determinaron mediante análisis inmunorradiométrico (Immunotech SA, Marsella, Francia, y Scantibodies ACTH kit, Scantibodies Laboratory, Santee, Estados Unidos, respectivamente).

También se determinaron los valores plasmáticos de transferrina, prealbúmina, albúmina y proteína ligada al retinol, mediante nefelometría (Beckman Instruments Co., Fullertton, Estados Unidos). Los valores de referencia tenidos en cuenta son los del laboratorio del hospital (tabla 1).

\section{Definiciones de las disfunciones neuroendocrinas}

En el presente estudio no se ha realizado evaluación neuroendocrina dinámica y los criterios considerados como indicativos de disfunción hipofisaria fueron:

- Eje gonadotrófico. En los varones, cuando se registraron valores de testosterona menores que el in-

\section{TABLA 1. Valores hormonales de referencia}

\begin{tabular}{|lc|}
\hline Folitropina (U/l) & \\
Varones & $1,5-21,5$ \\
Mujeres, fase folicular/fase & \\
$\quad$ luteínica/menopausia & $3,5-12,5 / 1,7-7,7 / 25,8-135$ \\
Lutropina (U/l) & $1,7-8,6$ \\
Varones & \\
Mujeres, fase folicular/fase & $2,4-12,6 / 1-11,4 / 7,7-58,5$ \\
$\quad$ luteínica/menopausia & \\
17-betaestradiol (pmol/l) & \\
Mujeres, fase folicular/fase & $46-607 / 161-774 /<201$ \\
luteínica/menopausia & $9,9-27,8$ \\
Testosterona (nmol/l), varones & $127-637$ \\
Prolactina ( $\mu \mathrm{gU} / \mathrm{l})$ & $98-456$ \\
Varones & $10-90$ \\
Mujeres & $171-536$ \\
Corticotropina (pg/ml) & $0,17-5,5$ \\
Cortisol matutino (nmol/l) & $9-28,4$ \\
Tirotropina $\mu \mathrm{U} / 1$ & $200-360$ \\
T4-libre (pmol/l) & $3.500-5.200$ \\
Transferrina (mg/dl) & $20-40$ \\
Albúmina (mg/dl) & $3-6$ \\
Prealbúmina (mg/dl) & $(\mu \mathrm{g} / \mathrm{ml})$ \\
Proteína ligada al retinol (mg/dl) & $230-390$ \\
Factor del crecimiento similar a la insulina & $75-310$ \\
<30 años & $125-310$ \\
$30-39$ años & $70-265$ \\
$40-49$ años & $95-270$ \\
$50-59$ años & \\
$60-65$ años & \\
&
\end{tabular}


TABLA 2. Características generales de severidad de pacientes con traumatismo craneoencefálico grave $(n=136)$

\begin{tabular}{|lc|}
\hline Edad (años) & $28(16-65)$ \\
Varones & $123(90,4 \%)$ \\
IMC & $24,69(18,3-37,6)$ \\
GCS & $7(4-8)$ \\
GCS [pacientes, n] & $4[6], 5[5], 6[22], 7[88], 8[15]$ \\
APACHE II & $13(7-29)$ \\
ISS & $29(16-59)$ \\
Alteraciones pupilares & $29(21,3 \%)$ \\
Diabetes insípida & $37(27,2 \%)$ \\
Traumatismo facial & $47(34,6 \%)$ \\
Traumatismo torácico & $26(19,1 \%)$ \\
Traumatismo abdominal & $15(11 \%)$ \\
Traumatismo de extremidades & $42(30,9 \%)$ \\
Traumatismo vertebral & $14(10,3 \%)$ \\
Traumatismo pelviano & $8(5,9 \%)$ \\
Uso de dopamina & $14(10,3 \%)$ \\
Uso de noradrenalina & $77(56,6 \%)$ \\
Muertes & $21(15,4 \%)$ \\
\hline
\end{tabular}

GCS: Glasgow Coma Scale; IMC: índice de masa corporal; ISS: Injury Severity Scale. Los valores expresan media (intervalo) o n (\%).

tervalo de normalidad, en presencia de valores normales o bajos de gonadotropinas. En las mujeres, cuando se registró un valor de 17-betaestradiol plasmático bajo según el momento del ciclo menstrual en las premenopáusicas y $<201 \mathrm{pmol} / 1$ en las posmenopáusicas, con valores normales o bajos de gonadotropinas.

- Eje tiroideo. Valor T4 libre $\leq 0,7 \mathrm{pmol} / \mathrm{l}$ con valores normales o bajos de TSH.

- Eje corticotropo. Concentraciones de cortisol basal $<171 \mathrm{nmol} / \mathrm{l}$ con valores normales o bajos de ACTH.

- Eje somatotropo. Se valoró la función del eje somatotropo determinando los valores basales de IGF-
1. Un valor de IGF-1 menor que el intervalo de normalidad correspondiente, según la edad del paciente, se consideró sospechoso de posible disfunción del eje somatotropo.

- Diabetes insípida. Cuando se registró un valor de sodio plasmático mayor que $150 \mathrm{mmol} / \mathrm{l}$, con poliuria mayor de 3 l/24 h y densidad urinaria menor de 1.005 .

\section{Análisis de los datos}

Las variables continuas se presentan con la mediana y sus correspondientes intervalos y la comparación entre grupos se realizó mediante el test de la U de Mann-Whitney. Las variables categóricas se analizaron mediante la prueba de la $\chi^{2}$. Los resultados se han considerado significativos para un valor de $\mathrm{p}=$ 0,05 . Todo el análisis se ha realizado con el paquete estadístico SPSS 12.0 para Windows.

\section{RESULTADOS}

En el presente estudio se incluyó a 136 pacientes con TCE graves. Las características generales de los pacientes estudiados quedan recogidas en la tabla 2. En cuanto a los mecanismos lesionales (fig. 1), los accidentes de circulación $(n=84,61,7 \%)$, sobre todo los de motocicletas $(n=50,36,7 \%)$, y la precipitación de altura $(n=34,25 \%)$ fueron los más frecuentes. Les siguen los atropellos a peatones $(5,2 \%)$, caídas $(4,5 \%)$ y otras $(3,6 \%)$. Las lesiones extracraneales concomitantes que encontramos se detallan en la tabla 2. La distribución del tipo de lesión cerebral, según los hallazgos tomográficos, se recogen en la figura 2. En nuestra serie encontramos que los factores en relación con lesión cerebral secundaria más frecuentes fueron hipertermia $(39 \%)$ y PPC baja (39\%),

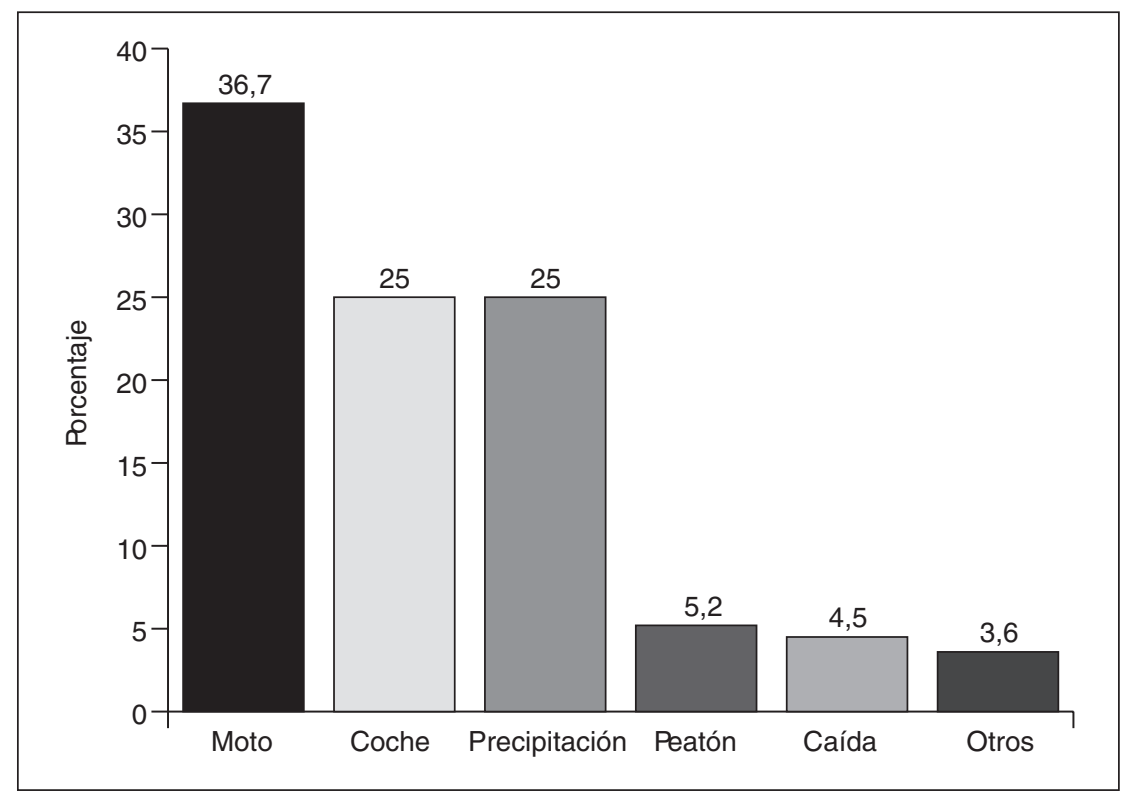

Figura 1. Mecanismos lesionales de los traumatismos craneoencefálicos graves. 


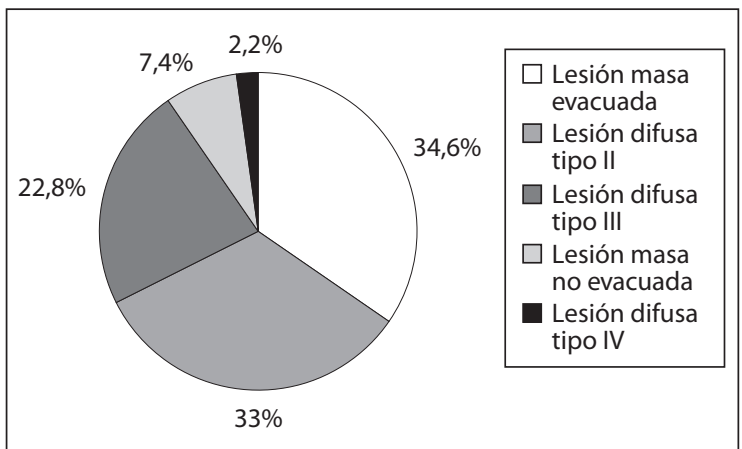

Figura 2. Tipo de lesión cerebral según la TCDB en los pacientes con traumatismo creaneoencefálico grave.

seguidos de hipotensión arterial $(30,1 \%)$, anemia $(27,9 \%), \operatorname{HEC}(22,7 \%)$ e hipoxia $(21,3 \%)$.

Las concentraciones de cada hormona hipofisaria y periférica en el tercer día tras la lesión traumática quedan recogidas en las tablas 3 y 4 , respectivamente. Hay dos hormonas cuyos patrones pueden aparecer elevados, como la ACTH y la PRL, que están elevadas en un 12,5 y un $38,5 \%$, respectivamente. Para estas dos hormonas los porcentajes de pacientes que presentan patrones elevados y descendidos son similares: el 12,5 frente al $11,8 \%$ para la ACTH y el 38,5 frente al 34,1\% para la PRL. Cuando estudiamos las hormonas periféricas, observamos que, tanto en varones como en mujeres, las que descendieron más frecuentemente fueron los esteroides sexuales; testosterona en un $91,7 \%$ de los varones y estradiol en un $66,7 \%$ de las mujeres. El estado nutricional, evaluado por las concentraciones plasmáticas de transferrina, prealbúmina, albúmina y proteína ligada al retinol, queda recogido en la tabla 5; se observa hipoproteinemia en la fase aguda de la enfermedad.

Respecto al estudio de disfunción neuroendocrina, encontramos que los pacientes que presentaron al menos un eje disfuncionante fueron 101 de 136 $(74,2 \%)$. De éstos, 79/136 (58\%) tuvieron afectación de un solo eje; $22 / 136(16,2 \%)$, de dos ejes, y no hubo ninguno que presentara tres o más ejes afectados. Desglosados por ejes, encontramos que presentaron alteración del eje gonadotropo $87(63,7 \%)$ pacientes; tirotropo, $12(8,8 \%)$ pacientes, y del eje corticotropo, $1(0,7 \%)$ paciente. En $90(66,7 \%)$ casos se registraron valores de IGF-1 por debajo del intervalo de normalidad para la edad del paciente, pero sólo $26(19,1 \%)$

TABLA 3. Concentraciones de hormonas hipofisarias al tercer día después de traumatismo craneoencefálico grave $(n=136)$

\begin{tabular}{|lc|}
\hline Baja lutropina & $68,9 \%$ \\
Baja folitropina & $61,5 \%$ \\
Baja prolactina & $38,5 \%$ \\
Baja tirotropina & $25 \%$ \\
Baja corticotropina & $11,8 \%$ \\
Alta prolactina & $34,1 \%$ \\
Alta corticotropina & $12,5 \%$ \\
\hline
\end{tabular}

TABLA 4. Concentraciones de hormonas periféricas al tercer día tras el traumatismo craneoencefálico grave $(n=136)$

\begin{tabular}{|lc|}
\hline Baja testosterona & $91,7 \%$ \\
Bajo estrógeno & $66,7 \%$ \\
Baja T4 & $44,1 \%$ \\
Bajo cortisol & $7,5 \%$ \\
Alto cortisol & $35,1 \%$ \\
Baja IGF-1 $(<200)$ & $66,1 \%$ \\
Baja IGF-1 $(<90)$ & $19,1 \%$ \\
\hline
\end{tabular}

IGF-1: factor de crecimiento similar a la insulina 1.

tenían valores plasmáticos de IGF-1 por debajo de 90 $\mathrm{ng} / \mathrm{ml}$, como dato de elevada sospecha de déficit de $\mathrm{GH}^{14}$ (fig. 3).

Cuando comparamos la diferencia en porcentajes de disfunción hormonal entre vivos y muertos, encontramos que 85 pacientes de los $115(73,9 \%)$ que sobrevivieron presentaron alteración al menos en un eje. Del grupo que fallecieron (21 pacientes), 16 $(76,2 \%)$ presentaron alteración en al menos un eje (p $=0,82$ ) (fig. 4).

\section{DISCUSIÓN Y CONCLUSIONES}

Nuestros resultados muestran que durante la fase inicial, tras sufrir un TCEG, hasta casi tres cuartas partes de los pacientes presentan disfunción neuroendocrina, con al menos un eje hipofisario afectado.

Hasta hace relativamente poco tiempo, la comunidad médica consideraba que el hipopituitarismo que sigue a un TCE era $\operatorname{raro}^{10}$. El primer caso de hipopituitarismo traumático fue descrito en 1914 por Cyran ${ }^{14}$. Escamilla et al $^{15}$, en 1944, publicaron un estudio de revisión de la literatura que evaluaba el hipopituitarismo patológico. Sólo en $4(0,7 \%)$ de 595 casos revisados la causa de hipopituitarismo fue el TCE. Posteriormente, otros estudios realizados por Altman et $\mathrm{al}^{16} \mathrm{y}$ Edwards et $\mathrm{al}^{17}$ describieron más casos. Sin embargo, el significado clínico de estas alteraciones endocrinas tras el TCE no ganaría importancia hasta el trabajo de Kelly et al ${ }^{18}$ quienes objetivaron que el $36 \%$ de la población estudiada (22 pacientes con TCE) presentaba alteraciones en la función endocrina cuando se le realizó prueba de provocación y un $22 \%$ tuvo alteraciones en dos de los componentes del eje hipotálamo-hipofisario. Otros trabajos que apoyan la mayor frecuencia de la afectación del eje hipotálamo-hipofisario son los de Benvenga et al ${ }^{19}$ (357 casos en 15 años) y Lieberman et $\mathrm{al}^{7}(68,5 \%)$. No obstante, todos estos trabajos ana-

\section{TABLA 5. Marcadores nutricionales en pacientes con traumatismo craneoencefálico grave $(n=136)$}

$\begin{array}{ll}\text { Baja albúmina } & 97,8 \% \\ \text { Baja transferrina } & 96,7 \% \\ \text { Baja prealbúmina } & 95,6 \% \\ \text { Baja proteína ligada al retinol } & 91,1 \%\end{array}$




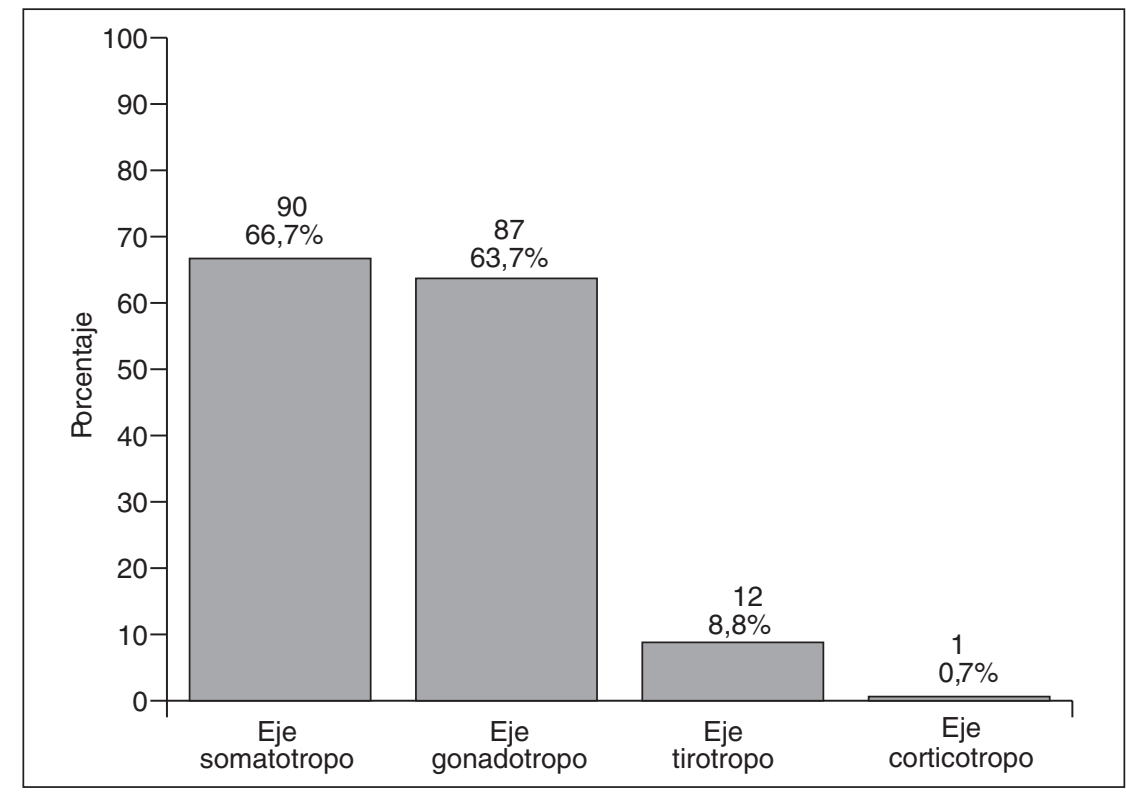

Figura 3. Disfunción neuroendocrina al tercer día en pacientes críticos con traumatismo craneoencefálico grave.

lizan principalmente a pacientes que han sufrido un TCE semanas o meses antes del estudio hormonal.

En 2004, un grupo de expertos ${ }^{20}$, reunidos para el análisis de las alteraciones endocrinas tras un TCE, recomendaba la realización de estudios prospectivos que incluyeran a pacientes con TCE en la fase aguda y en los primeros 12 meses tras el traumatismo. Cuando se revisan los estudios existentes en la literatura, son pocos los artículos originales realizados en la primera semana tras el episodio traumático ${ }^{21,22}, \mathrm{y}$ son muchos los que realizan la evaluación pasada la primera semana del evento ${ }^{23-27}$. Por eso queremos destacar la importancia del presente estudio, al ser el mayor trabajo prospectivo realizado para evaluar de forma sistemática la frecuencia de las alteraciones del

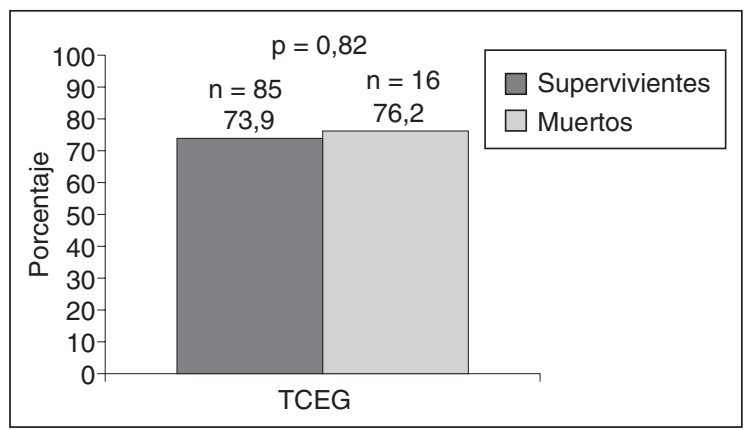

Figura 4. Supervivencia y mortalidad de los pacientes tras traumatismo craneoencefálico grave (TCEG) que presentaron alteración de al menos un eje hipotálamo-hipofisario. De los pacientes con TCEG, 85 (73,9\%) de los 115 que sobrevivieron y 16 (76,2\%) de los 21 fallecidos tras el TCEG presentaron alteración de al menos un eje hipotálamo-hipofisario. No hay diferencia estadísticamente significativa entre los pacientes fallecidos y los supervivientes con alteración de al menos un eje hipotálamo-hipofisario. eje hipotálamo-hipofisario en la fase aguda de pacientes con TCEG. Por otra parte, la variabilidad en los resultados de los distintos estudios se debe a la metodología de la selección de la muestra para la determinación hormonal. En nuestro estudio se realizó al tercer día del traumatismo por considerar ese momento el más idóneo tras el período inicial de reanimación y estabilización.

Nuestros resultados han mostrado que casi tres cuartas partes de los pacientes que sufren un TCEG presentan criterios indicativos de disfunción hipofisaria en el tercer día de evolución.

Al comparar nuestros resultados con los existentes en la literatura, observamos que el trabajo de Cernak et $\mathrm{al}^{22}$ evidenció significativas alteraciones neuroendocrinas en pacientes que sufrieron un TCE directo o indirecto, ya que registraron alteraciones en los ejes tirotropo, gonadotropo y corticotropo. Bondanelli et $\mathrm{al}^{4}$ realizaron una revisión de los principales trabajos que estudian el eje hipotálamo-hipofisario a partir del primer mes del TCE, valorando a 344 pacientes con distintos grado de severidad de TCE. Aunque no es comparable con el presente trabajo, por las diferencias metodológicas, encontramos elementos coincidentes; un hallazgo frecuente en los TCE es la afectación de uno o dos ejes, es bastante improbable la afectación de todos los ejes y predomina la afectación de un solo eje neuroendocrino. Si confrontamos nuestro trabajo con otros que evalúan la respuesta neuroendocrina en pacientes no traumatizados ${ }^{28}$ y traumatizados sin lesión craneal ${ }^{21,22}$, observamos que también hay alteraciones neuroendocrinas. Sin embargo, las diferencias metodológicas hacen que los resultados no sean comparables con los nuestros.

Una clara debilidad del presente estudio ha sido la valoración de la función somatotrópica, que se reali- 
zó únicamente mediante la determinación plasmática de IGF-1. La valoración de la función somatotrópica ha sido un aspecto controvertido; clásicamente, el test de tolerancia a la insulina es la prueba de referencia más aceptada ${ }^{29}$, aunque las complicaciones de esta prueba, como las crisis convulsivas, han hecho que más recientemente se hayan desarrollado pruebas dinámicas con el factor de liberación de la hormona del crecimiento ${ }^{30,31}$. Sin embargo, la realización de este tipo de pruebas en la fase inicial de un TCEG puede ser complicada, por lo que la determinación de IGF-1 podría tener un valor indicativo de los pacientes con posible déficit de $\mathrm{GH}$, principalmente con valor de IGF-1 $<90 \mathrm{ng} / \mathrm{ml}^{14}$. No obstante, la determinación de IGF-1 también plantea problemas como indicador de la secreción o la acción de la hormona del crecimiento en la fase aguda de la enfermedad, ya que Agha et $\mathrm{al}^{32}$ no han registrado diferencias significativas en las concentraciones plasmáticas de IGF-1 entre pacientes deficientes o no de $\mathrm{GH}$ en la fase aguda del TCE.

En cuanto a la mortalidad, el porcentaje de pacientes con alteración de al menos un eje hipotálamo-hipofisario alterado fue mayor entre los fallecidos que entre los que sobrevivieron al TCEG, aunque esta diferencia no es significativa estadísticamente.

Aunque nuestros resultados corroboran los hallazgos de la elevada frecuencia de disfunción hipofisaria en la fase aguda del TCEG, no permiten aclarar si dicha disfunción es secundaria a lesiones estructurales por el traumatismo o si se trata de una respuesta encuadrada en la repuesta fisiológica a los procesos críticos agudos. La importancia de las alteraciones neuroendocrinas en el desarrollo de la enfermedad, así como el papel que tiene en la evolución y la recuperación de estos pacientes, está por dilucidar. Estudios encaminados a valorar la función hipofisaria en pacientes con TCEG y pacientes traumatizados sin TCE tal vez puedan ayudar a clarificar esta cuestión. Adicionalmente, el hecho de que las alteraciones endocrinas hipofisarias puedan ser transitorias hace que el papel del tratamiento hormonal en la fase aguda del TCE también sea una cuestión pendiente de clarificar con estudios adicionales ${ }^{33}$. No obstante, a pesar de estas incertidumbres sobre el significado y el manejo de la disfunción hipofisaria en la fase aguda del TCEG, nuestros resultados apoyan la necesidad de evaluar la función hipofisaria de estos pacientes como primer paso en el seguimiento endocrinológico a largo plazo, ya que estas alteraciones pueden persistir y constituirse en un factor de limitación en la calidad de vida de los supervivientes.

\section{BIBLIOGRAFÍA}

1. Marshall LF, Gautille T, Klauber MR, Eisenberg HM, Jane JA, Luerssen TG, et al. The outcome of severe closed head injury. J Neurosurg. 1991;75 Suppl:28-36.

2. Maas A, Dearden M, Servadei F, Stocchetti N, Unterberg A. Current recommendations for neurotrauma. Curr Opin Crit Care. 2000;6:281-92.
3. Muñoz-Céspedes JM, Paúl Lapedriza N, Peñegrín Valero C, Tirapu Ustarroz J. Factores de pronóstico en los traumatismos craneoencefálicos. Rev Neurol. 2001;32:351-64.

4. Bondanelli M, Ambrosio MR, Zatelli MC, De Marinis L, Degli Uberti EC. Hypopituitarism after traumatic brain injury. Eur J Endocrinol. 2005;152:679-91.

5. Junqué C. Secuelas neuropsicológicas de los traumatismos craneoencefálicos. Rev Neurol. 1999;28:423-9.

6. Woolf PD. Hormonal responses to trauma. Crit Care Med. 1992;20:216-26.

7. Lieberman SA, Oberoi AL, Gilkison CR, Masel BE, Urban RJ. Prevalence of neuroendocrine dysfunction in patients recovering from traumatic brain injury. J Clin Endocrinol Metab. 2001;86:2752-6.

8. Bondanelli M, De Marinis L, Ambrosio MR, Monesi M, Valle D, Zatelli MC, et al. Ocurrence of pituitary dysfunction following traumatic brain injury. J Neurotrauma. 2004;21:685-96.

9. Aimaretti G, Ambrosio MR, Di Somma C, Fusco A, Cannavo $\mathrm{S}$, Gasperi M, et al. Traumatic brain injury and subarachnoid haemorrhage are conditions at high risk for hypopituitarism: screening study at 3 months after brain injury. Clin Endocrinol (Oxf). 2004;61:320-6.

10. Elie P, Elovic MD. Anterior pituitary dysfunction after traumatic brain injury, Part I. J Head Trauma. 2003;18:541-3.

11. Masel BE. Rehabilitation and hypopituitarism after traumatic brain injury. Growth Horm IGF Res. 2004;14 Suppl:108-13.

12. Brain Trauma Foundation. Critical pathway for the treatment of established intracranial hypertension. J Neurotrauma. 1996;13:719-20.

13. Marshall LF, Marshall SB, Klauber MR, Van Berkum Clark M, Eisenberg HM, Jane JA, et al. A new classification of head injury based on computarized tomography. J Neurosurg. 1991;75 Suppl:14-20.

14. Cyran E. Hypophysenschädigung durch schädelbasisfraktur. Dtsch Med Wschr. 1918;44:1261.

15. Escamilla RF, Lisser H. Simmonds disease. J Clin Endocrinol. 1942;2:65-96.

16. Altman R, Pruzansski W. Post-traumatic hypopituitarism. Ann Intern Med. 1961;55:149-54.

17. Edwards OM, Clack JDA. Post-traumatic hypopituitarism. Six cases and revision of the literature. Medicine. 1986;65:281-90.

18. Kelly DF, Gaw Gonzalo IT, Cohan P, Berman N, Swerdloff $R$, Wang C. Hypopituitarism following traumatic brain injury and aneurysmal subarachnoid hemorrhage: a preliminary report. J Neurosurg. 2000;93:743-52.

19. Benvenga S, Campeen A, Ruggeri RM, Trimachi F. Hypopituitarism secondary to head trauma. J Clin Endocrinol Metab. 2000;85:1353-61.

20. Casanueva FF, Ghigo E, Popovic V, and the Athens TBI and Hypopituitarism Study Group. Hypopituitarism following traumatic brain injury (TBI): A guideline decalogue. J Endocrinol Invest. 2004;27:793-5.

21. Chiolero R, Schutz Y, Lemarchand T, Felber JP, De Tribolet $\mathrm{N}$, Freeman J, et al. Hormonal and metabolic changes following severe head injury or noncranial injury. J Parenter Enteral Nutr. 1989;13:5-12.

22. Cernak I, Savic VJ, Lazarov A, Joksimovic M, Markovic S. Neuroendocrine response following graded traumatic brain injury in male adults. Brain Inj. 1999;13:1005-15.

23. Piek J, Chesnut RM, Marshall LF, Van Berkum Clark M, Klauber MR, Blunt BA, et al. Extracranial complications of severe head injury. J Neurosurg. 1992;77:901-7.

24. Agha A, Rogers B, Sherlock M, O'Kelly P, Tormey W, Phillips J, et al. Anterior pituitary dysfunction in survivors of traumatic braun injury. J Clin Endocrinol Metab. 2004;89:4929-36.

25. Urban RJ, Harris P, Masel BE. Anterior hypopituitarism following traumatic brain injury. Brain Inj. 2005;19:349-58.

26. Schneider M, Schneider HJ, Stalla GK. Anterior pituitary hormone abnormalities following traumatic brain injury. J Neurotrauma. 2005;22:937-46.

27. Popovic V, Aimaretti G, Casanueva FF, Ghigo E. Hypopituitarism following traumatic brain injury. Front Horm Resp. 2005;33:33-44.

28. Van den Gerghe G. Novel insights into the neuroendocrinology of critical illness. Eur J Endocrinology. 2000;143:1-13. 
29. Consensus guidelines for the diagnosis and treatment of adults with growth hormone deficiency: Summary statement of the Growth Hormone Research Society Workshop on Adult Growth Hormone Deficiency. J Clin Endocrinol Metab. 1998;83:379-81.

30. Cordido F, Alvarez-Castro P, Isidro ML, Casanueva FF, Dieguez C. Comparison between insulin tolerance test, growth hormone (GH)-releasing hormone (GHRH), GHRH plus acipimox and GHRH plus GH-releasing peptide- 6 for the diagnosis of adult GH deficiency in normal subjects, obese and pituitary patients. Eur J Endocrinol. 2003;149:117-22.
31. Leal-Cerro A, Flores JM, Rincón MD, Murillo F, Pujol M, Garcia-Pesquera F, et al. Prevalence of hypopituitarism and growth hormone deficiency in adults long-term after severe traumatic brain injury. Clin Endocrinol (Oxf). 2005;62:525-32.

32. Agha A, Rogers B, Mylotte D, Taleb F, Tormey W, Phillips $\mathrm{J}$, et al. Neuroendocrine dysfunction in the acute phase of traumatic brain injury. Clin Endocrinol (Oxf). 2004;60:584-91.

33. Cohan P, Wang C, McArthur DL, Cook SW, Dusick JR, Armin B, et al. Acute secondary adrenal insufficiency after traumatic brain injury: A prospective study. Crit Care Med. 2005; 33:2358-66. 